\title{
SOLAR IRRADIANCE VARIABILITY AND LUMINOSITY CHANGES
}

\author{
PETER FOUKAL \\ Cambridge Research and Instrumentation, Inc \\ Cambridge, Massachusetts, USA
}

This review presents a discussion of radiometric measurements of solar total irradiance variation, $\Delta S$, on time scales of days, to the 15-year extent of present measurements. It does not cover variations in spectral irradiance, particularly the relatively large solar UV and EUV flux variations of great importance in studies of trends in stratospheric ozone, and of upper atmospheric heating variations.

Analysis of the pyrheliometry of $\Delta S$ carried out from the Solar Maximum Mission, Nimbus-7 and ERBS satellites has shown that a) the sun brightens by $0.05-0.1 \%$ around activity maximum and; b) it dims by up to approximately $0.2 \%$ when large sunspot groups cross the disc. Empirical models of $\Delta \mathrm{S}$ based on areas and photometric contrasts of sunspots, and on proxies of facular area, are remarkably successful in matching the observed record of $\Delta \mathrm{S}$, on time scales between days and the full 15- year record available since 1978 . These models indicate that the opposite contributions of sunspots and faculae in active regions roughly balance over time scales of years, and the net solar brightening observed around peak activity is caused by increased emission from the photospheric magnetic network.

Discrepancies exist between the model and measurements, near the peaks of both cycles 21 and 22. It is not yet clear whether these are caused by pyrheliometry measurement errors, or by inadequacies of the model. But the discrepancies arise at the two times when the pyrheliometry record is least certain.

The most plausible physical explanation of the sunspot-induced decreases in total irradiance is in terms of photospheric heat flow blocking by the sunspot magnetic field. Both analytical and numerical calculations have been performed, of the transient effect of a "thermal plug" placed at the photospheric surface. These calculations are able to reproduce the depth, duration, and shape of the irradiance response to an observed change in sunspot projected area, given reasonable estimates of the heat diffusivity 
of the solar convection zone layers around the spot. Similar arguments can be used to reproduce the influence of faculae upon total irradiance (and luminosity).

Photometric searches have been carried out for evidence of photospheric heat flow inhomogeneities outside of active regions. But no convincing signal (from e.g. thermal shadows or large-scale convective structures) has yet been identified down to the noise level of $\Delta \mathrm{T}_{b} \sim 0.2 \mathrm{~K}$ set by the noise of granulation and 5-minute oscillations. New information on global $\Delta \mathrm{T}_{e f f}$ may emerge from analysis of time variations in the frequencies of moderateand low-l solar p-mode oscillations. The presence of such variations in correlation with the 11-year cycle, has been demonstrated. Further analysis will show whether they can be used to separate the magnetically-induced variations of $\Delta \mathrm{S}$ and $\Delta \mathrm{L}$, from variations in $\Delta \mathrm{T}_{\text {eff }}$ caused by e.g. global variations of convective efficiency.

The most important issue at the frontier of solar luminosity studies is whether slow variations of $\Delta \mathrm{L}$ and $\Delta \mathrm{S}$ can occur, correlated with the envelope of activity, but of larger amplitude than those observed to be caused by photospheric magnetic structures, over the past 15 years of direct pyrheliometry. An answer is required, to decide whether a solar luminosity mechanism can explain the remarkably good correlation of the solar activity envelope, and the Earth's temperature record over the past millennium.

Interesting evidence for the possibility of past and future solar luminosity variations in the $0.5-1 \%$ range has been presented from analysis of photometry of stars similar in mass and age to the sun. The key issue here is whether these solar-like stars are in fact sufficiently similar to the sun for the luminosity variation mechanisms operating on them, to operate on the sun in the relatively recent past, or foreseeable future.

We must always keep in mind that, while solar luminosity variations provide the most direct link between solar activity and climate, further research may yet show that more complex couplings, driven by variable solar UV or particle fluxes, may prove dominant in our continuing efforts to understand the relationship between changes in the sun and the troposphere. 